\title{
Near-Field Imaging of Phased Array Metasurfaces
}

\section{Citation}

Bohn, Bernhard J., Martin Schnell, Mikhail A. Kats, Francesco Aieta, Rainer Hillenbrand, and Federico Capasso. 2015. "Near-Field Imaging of Phased Array Metasurfaces." Nano Letters 15 (6): 3851-58. https://doi.org/10.1021/acs.nanolett.5b00692.

\section{Permanent link}

http://nrs.harvard.edu/urn-3:HUL.InstRepos:41371320

\section{Terms of Use}

This article was downloaded from Harvard University's DASH repository, WARNING: This file should NOT have been available for downloading from Harvard University's DASH repository.

\section{Share Your Story}

The Harvard community has made this article openly available.

Please share how this access benefits you. Submit a story.

Accessibility 


\title{
Near-Field Imaging of Phased Array Metasurfaces
}

\author{
Bernhard J. Bohn, ${ }^{\dagger, \ddagger}$ Martin Schnell, ${ }^{\S}$ Mikhail A. Kats, ${ }^{*}{ }^{\dagger,}, \|$ Francesco Aieta, ${ }^{\dagger}$ Rainer Hillenbrand, ${ }^{\#, \perp}$ \\ and Federico Capasso ${ }^{\dagger}$
}

\author{
${ }^{\dagger}$ School of Engineering and Applied Sciences, Harvard University, Cambridge, Massachusetts 02138, United States \\ ${ }^{\ddagger}$ Fakultät für Physik, Ludwig-Maximilians-Universität, 80799 München, Germany \\ ${ }^{\S}$ CIC nanoGUNE, 20018 Donostia, San Sebastian, Basque Country, Spain \\ ${ }^{\|}$Department of Electrical and Computer Engineering, University of Wisconsin - Madison, Madison, Wisconsin 53706, United States \\ ${ }^{\#}$ CIC nanoGUNE and EHU/UPV, 20018 Donostia, San Sebastian, Basque Country, Spain \\ ${ }^{\perp}$ IKERBASQUE, Basque Foundation for Science, 48011 Bilbao, Basque Country, Spain
}

\section{Supporting Information}

ABSTRACT: Phased-antenna metasurfaces can impart abrupt, spatially dependent changes to the amplitude, phase, and polarization of light and thus mold wavefronts in a desired fashion. Here we present an experimental and computational near-field study of metasurfaces based on near-resonant V-shaped antennas and connect their near- and far-field optical responses. We show that far fields can be obtained from limited, experimentally obtained knowledge of the near fields, paving the way for experimental near-field characterization of metasurfaces and other optical nanostructures and prediction of their far fields from the near-field measurements.

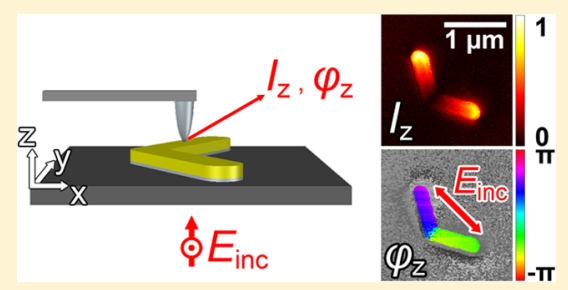

KEYWORDS: Optical antennas, metasurfaces, SNOM, near field, plasmonics

$\mathrm{V}$ arious widely used optical components such as lenses, prisms, and wave plates rely on the gradual change of phase and polarization of the light as it propagates through some material. ${ }^{1}$ As nanofabrication technologies have made it easier to generate structures with length scales smaller than the wavelength of light, new approaches to controlling optical wavefronts have emerged. One such approach involves metasurfaces, which consist of a metallic and/or dielectric patterned layer (or several layers) with thicknesses much smaller than the wavelength of light. The term "flat optics" is sometimes used to describe these quasi-two-dimensional structures. ${ }^{1,2}$ Metasurfaces with spatially varying optical response have enabled the observation of new optical phenomena such as anomalous reflection and refraction ${ }^{3}$ and direct coupling to surface waves, ${ }^{4}$ as well as the development of ultrathin optical components such as planar lenses, ${ }^{5-7}$ spiral phase plates, ${ }^{8}$ and wave plates. ${ }^{9}$

A widely studied metasurface comprises metallic V-shaped antennas which support symmetric and antisymmetric charge oscillation eigenmodes that can be selectively excited by the two linear polarizations of light (Figure 1a). By combining the scattering properties of these two modes, a single $\mathrm{V}$-antenna can be designed to impart a phase shift between the incident and scattered light in the range from 0 to $2 \pi .^{3,10}$ As a result these antennas can be tiled to create a metasurface which modulates the wavefront of light in a desired fashion. These metasurfaces are often used in cross-polarization mode (e.g., ref 3 ), but other modes of operation are possible., ${ }^{1,9}$

Since the introduction of these structures, there have been numerous studies which use metasurfaces for far-field control (e.g., refs 1-12) but little work to explore their near-field properties. Here we characterized the near-field response of individual, isolated $\mathrm{V}$-antennas around resonance using phaseresolved scattering-type scanning near-field optical microscopy (s-SNOM) and compared the experimental results with numerical simulations. We then examined the near field of an antenna array which imparts a linear phase gradient onto the incident light, resulting in anomalous reflection and refraction in the far field. ${ }^{3}$ We thus established a connection between the near- and far-field behavior of metasurfaces.

As viewed from the top, our $\mathrm{V}$-antennas consist of two gold rectangles with length $L$ and width $W$ which are connected at the center of one end at an angle $\Delta$ (Figure 1a). At the end of each rectangle is a semicircle of radius $r=W / 2$. We chose $\mathrm{V}$ antenna geometries such that our present results can be directly compared to existing far-field experiments in the literature. ${ }^{3}$ In particular we studied four different types of $\mathrm{V}$-antennas with opening angles $\Delta=60^{\circ}, 90^{\circ}, 120^{\circ}$, and $180^{\circ}$. A V-antenna supports two fundamental charge-oscillation eigenmodes (symmetric and antisymmetric). The symmetric mode is excited when the incident light is polarized along the symmetry axis $\hat{\mathbf{s}}$ of the antenna, while the antisymmetric mode is excited by light polarized along $\mathbf{a}$, which is orthogonal to $\hat{\mathbf{s}}$ (Figure 1a). We first characterized the fundamental first-order resonances in the near field for both modes of isolated $\mathrm{V}$-antennas which are the building blocks of our metasurfaces.

Received: February 19, 2015

Revised: $\quad$ May 7, 2015 
a
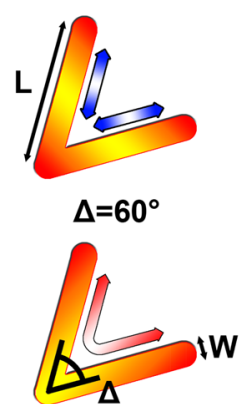

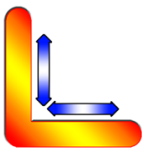

$90^{\circ}$

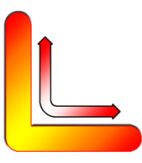

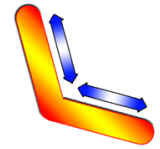

$120^{\circ}$

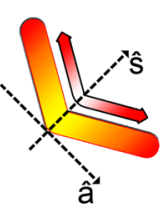

b

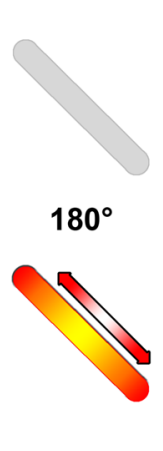

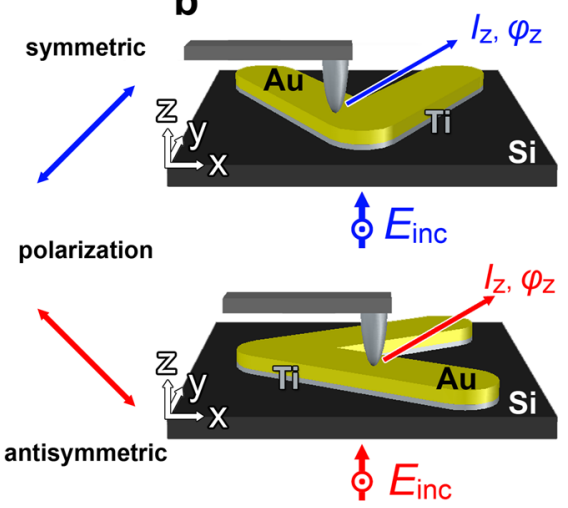

Figure 1. (a) Schematic of the V-antennas studied in this work. Each antenna is defined by its opening angle $\Delta$, arm length $L$, and width $W$. The symmetric charge-oscillation eigenmode is excited by light polarized along the symmetry axis of the $\mathrm{V}$-antenna $\hat{\mathbf{s}}$, and the antisymmetric mode by the orthogonal polarization (along â). The thick arrows with a color gradient indicate the direction of the current flow in each eigenmode while darker colors represent higher average charge density. The $180^{\circ}$ (linear) antenna features only one fundamental eigenmode. (b) Schematic of s-SNOM antenna mapping. The antennas are illuminated from the substrate side at normal incidence with a $y$-polarized $\mathrm{CO}_{2}$ laser beam $(\lambda=9.3 \mu \mathrm{m})$. The symmetric (top sketch, blue) and antisymmetric antenna modes (bottom sketch, red) are excited by aligning the antennas such that $\hat{s}$ and $\hat{a}$ are parallel to the polarization of the incident light, respectively. A dielectric tip locally scatters the antenna's near field. Interferometric detection of the scattered light yields the vertical near-field component $E_{z}$ and enables calculation of the near-field intensity $I_{z}=\left|E_{z}\right|^{2}$ and the near-field phase $\varphi_{z}=$ $\arg \left(E_{z}\right)$ (see Methods section for more details). A full diagram of our s-SNOM setup can be found in ref 35 .

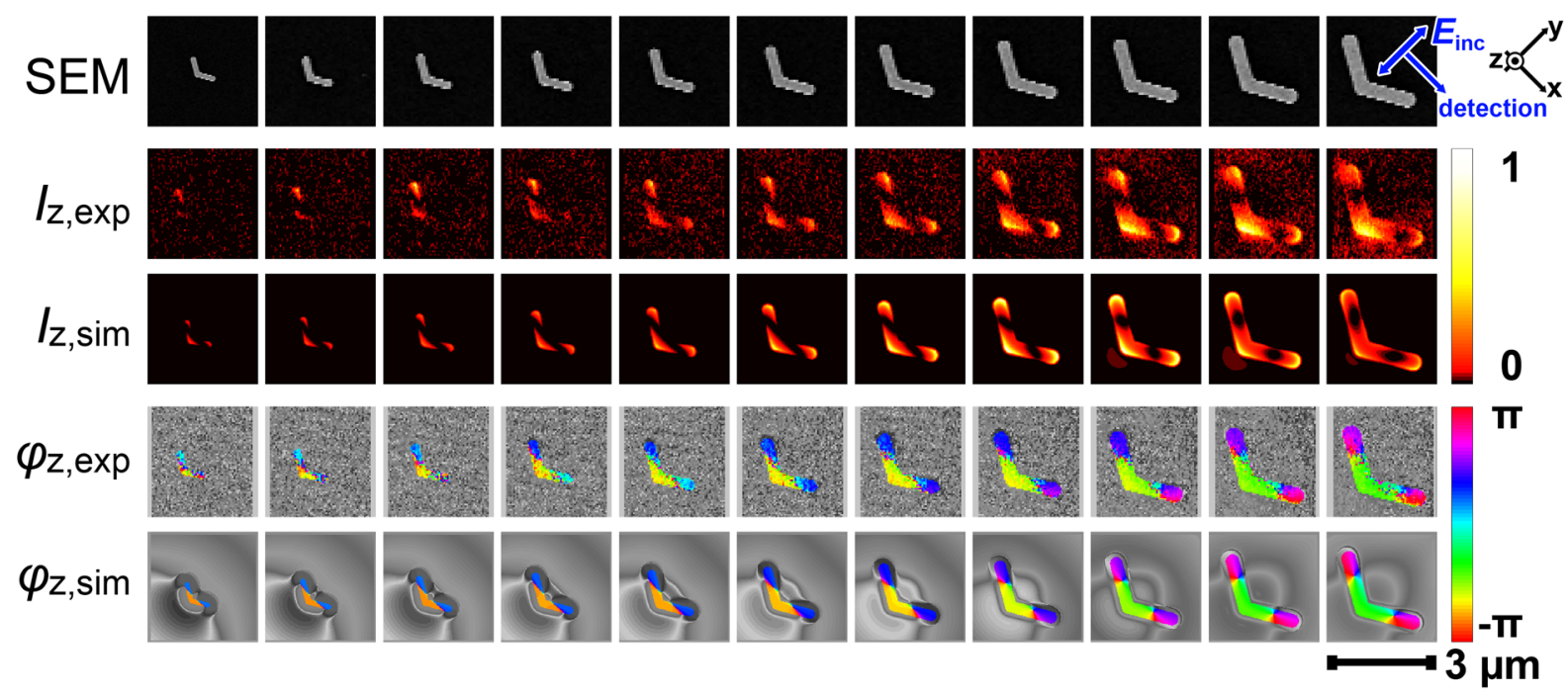

Figure 2. Experimental (exp) and simulated (sim) near-field maps of the symmetric mode of the $\Delta=120^{\circ}$ antennas for different antenna sizes (polarization of the incident light $E_{\text {inc }}$ and orientation indicated in the top right panel): scanning electron microscope (SEM) images, near-field intensity, and phase for the $z$-oriented electric field component. Both the calculations and the experimental measurements show that the near-field phase varies as a function of antenna size with most of the variation occurring when the antenna is near resonance, where the intensity reaches its maximum. We use a grayscale filter in the phase plots to de-emphasize the area not directly above the antennas.

The conventional way to probe an antenna resonance is to look at the response of some quantity (e.g., near-field intensity) for a single antenna as a function of wavelength. ${ }^{13}$ Because of experimental limitations, we instead pursued a complementary approach where the wavelength is fixed $(\lambda=9.3 \mu \mathrm{m})$, measuring a series of isolated antennas of varying sizes. ${ }^{14-17}$ This is roughly equivalent to measuring the wavelengthdependent spectrum of a single antenna due to the scale invariance of Maxwell's equations and the fact that at midinfrared wavelengths gold behaves similarly to a perfect electric conductor, $^{18}$ and thus its dispersion can be ignored. We scaled the length $L$ and width $W$, maintaining the thickness constant since it does not change the resonance frequency significantly (see Supporting Information, Section 4). Eleven antenna sizes were chosen for each opening angle $\Delta$, and the arm length $L$ and width $W$ increase in equidistant steps from the smallest to the biggest antenna, such that the ratio $L / W$ is kept constant. The range of sizes was chosen such that the resonances for both modes are observed in the experiment.

Using electron beam lithography with lift-off, the $50 \mathrm{~nm}$ thick $\mathrm{V}$-antennas were fabricated with gold $(\mathrm{Au})$ on top of a silicon ( $\mathrm{Si}$ ) substrate with a $5 \mathrm{~nm}$ titanium (Ti) adhesion layer in between (Figure $1 \mathrm{~b}$ ). We utilized s-SNOM in transmission mode, ${ }^{19-21}$ which provides amplitude- and phase-resolved nearfield mapping. By combining experimental s-SNOM measurements with finite-difference time-domain (FDTD) simulations of the antennas, we gained access to detailed information about the charge distribution at, around, and away from resonance. For this study we considered the intensity $I_{z}=\left|E_{z}\right|^{2}$ and the phase $\varphi_{z}=\arg \left(E_{z}\right)$ of the vertical near-field component $E_{z}$ (see 


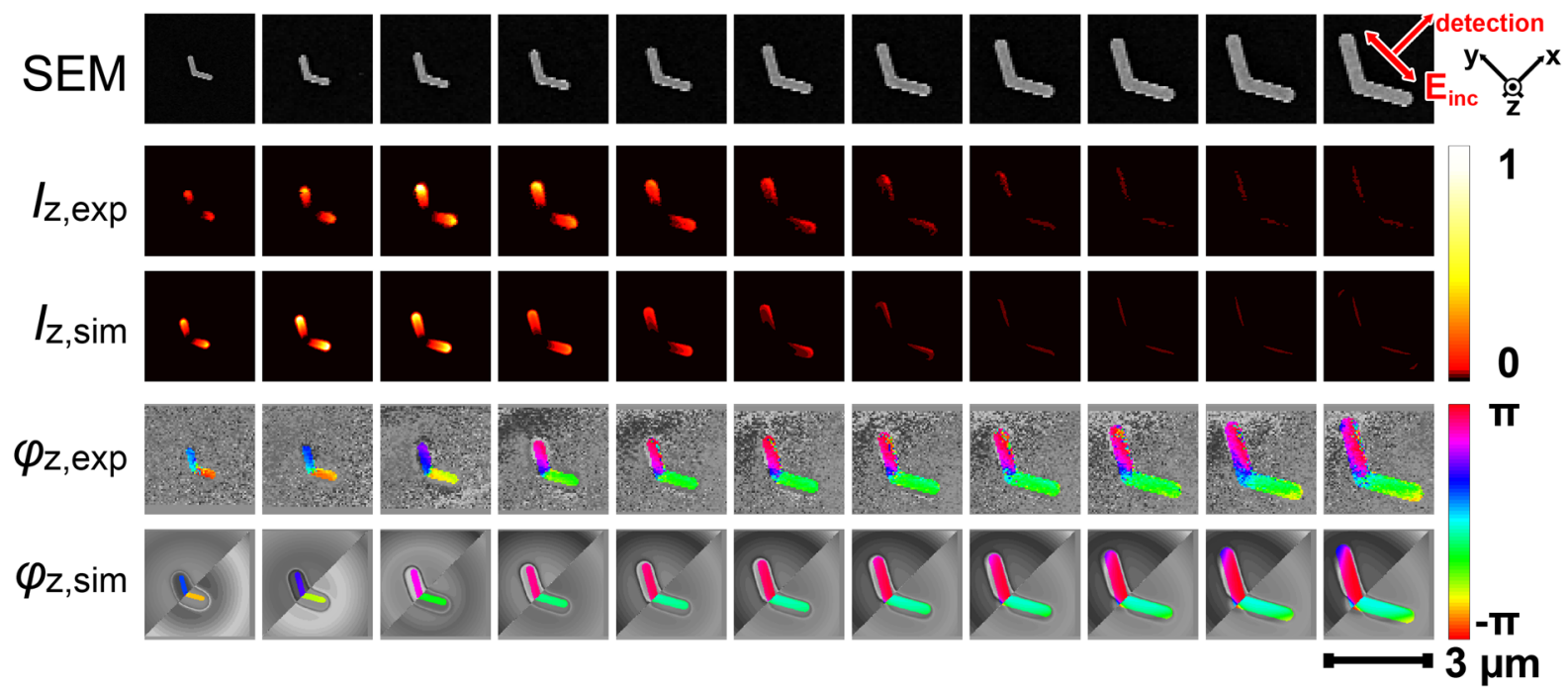

Figure 3. Experimental and simulated intensity and phase maps as described in Figure 2 for the antisymmetric mode of the $\Delta=120^{\circ}$ antennas.

Methods section for details). Due to space limitations, we only present the symmetric and antisymmetric modes of the $\Delta=$ $120^{\circ}$ antennas in the main text; the results for all other values of $\Delta$ can be found in the Supporting Information (Section 1).

Figure 2 shows the experimental and simulated intensity and phase maps of the near field for the symmetric mode, while Figure 3 shows the antisymmetric mode. The experimental data for the 11 antennas of the same shape, but increasing size is obtained in a single s-SNOM scan, thus allowing for a direct comparison of near-field intensity and phase between the antennas. On the sample each antenna is $5 \mu \mathrm{m}$ away from its neighbors to minimize coupling without making the overall scan area too large. For visual clarity, the intensity plots in Figures 2 and 3 are normalized to the highest intensity value. For the symmetric mode (Figure 2) both of the tips and the vertex of each $\mathrm{V}$-antenna are bright, while in the antisymmetric mode (Figure 3) the vertex stays dark, indicating no field buildup at the center. This makes it easy to distinguish between those two modes. The last two rows show the near-field phase above the antenna surface in the experiment $\left(\varphi_{z, \exp }\right)$ and the simulations $\left(\varphi_{\mathrm{z}, \mathrm{sim}}\right)$.

For a detailed analysis of the resonance behavior of the antennas measured in Figures 2 and 3, we assigned an averaged intensity value $I_{z, \text { avg }}$ to each antenna in order to compare the near-field enhancement for the different antenna sizes (Figure 4a). This quantity is the average value of all the intensity data points directly above one individual $\mathrm{V}$-antenna. We also read out the phase at the left tip of each antenna to observe the variation of the phase response across the resonance (Figure 4b) (see Methods section for details on visualization and analysis). The resonance of the antisymmetric mode (red curves) occurs for $L \approx 0.65 \mu \mathrm{m}$ and for the symmetric mode (blue curves) for $L \approx 1.3 \mu \mathrm{m}$, as can be appreciated from the near-field intensity plots (Figure $4 \mathrm{a}$ ). This factor of $\sim 2$ between the resonance wavelength of the symmetric and antisymmetric modes is expected since the current travels approximately twice the length per optical cycle in the antisymmetric mode (Figure 1a). From below resonance to above resonance the near-field phase varies by approximately $\pi .^{3}$ Figure 4 shows that, by changing the size of the antenna, a specific phase value can be selected, which enables the creation of antenna-based metasurfaces as discussed below.
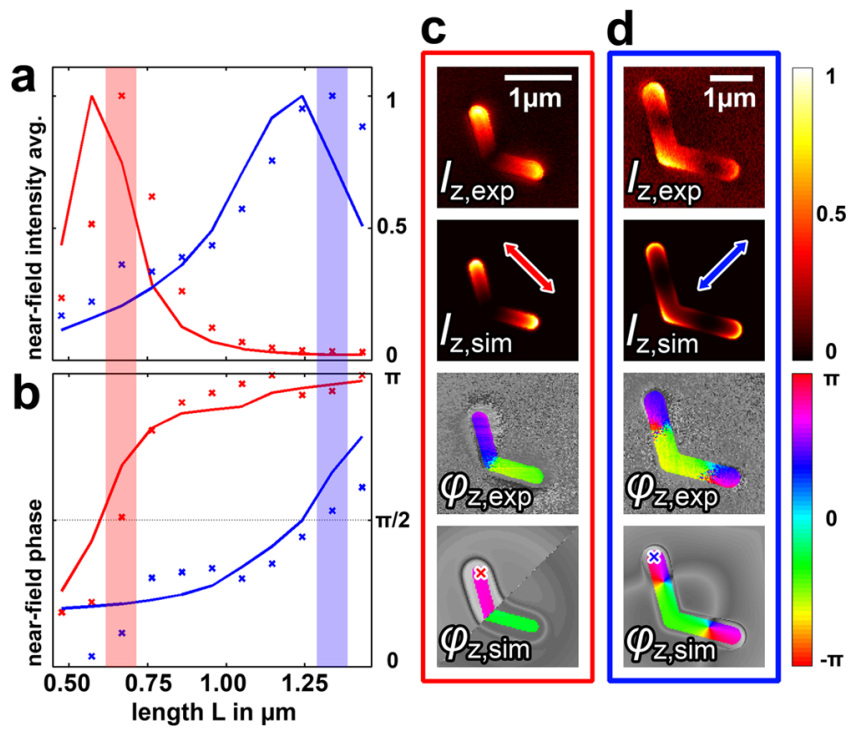

Figure 4. Plots of the averaged near-field intensity $I_{z, a v g}$ (a) and phase $\varphi_{z}$ (b) for the $\Delta=120^{\circ}$ antenna antisymmetric mode (red) and symmetric mode (blue) as a function of antenna size, represented by the arm length $L$. Simulation values are plotted as a solid line while crosses indicate the experimental values. For each 11-antenna series, the intensities are normalized to 1 . For each mode the antenna closest to resonance was remeasured with high resolution $(128 \times 128$ points $)$. The two transparent red and blue bars highlight these two antennas in plots (a) and (b). These additional measurements are presented in (c) and (d) for the antisymmetric mode and the symmetric mode, respectively. The phase values in (b) are taken at the position indicated by the cross in (c) and (d).

For each measurement series, we acquired high-resolution near-field images of the resonant antisymmetric and symmetric modes. In Figure 4c,d, we show the high-resolution images of the $120^{\circ} \mathrm{V}$-antenna, while images of the other antennas are included in the Supporting Information (Figures S2, S4, and S6). The measurements agree well with FDTD calculations, confirming that transmission-mode s-SNOM allows for reliable mapping of two-dimensional antenna structures.

The minor discrepancies in Figure $4 a, b$ between the simulation and experimental data of the symmetric mode (blue plots) for arm lengths $L<1 \mu \mathrm{m}$ are attributed to a small 
a
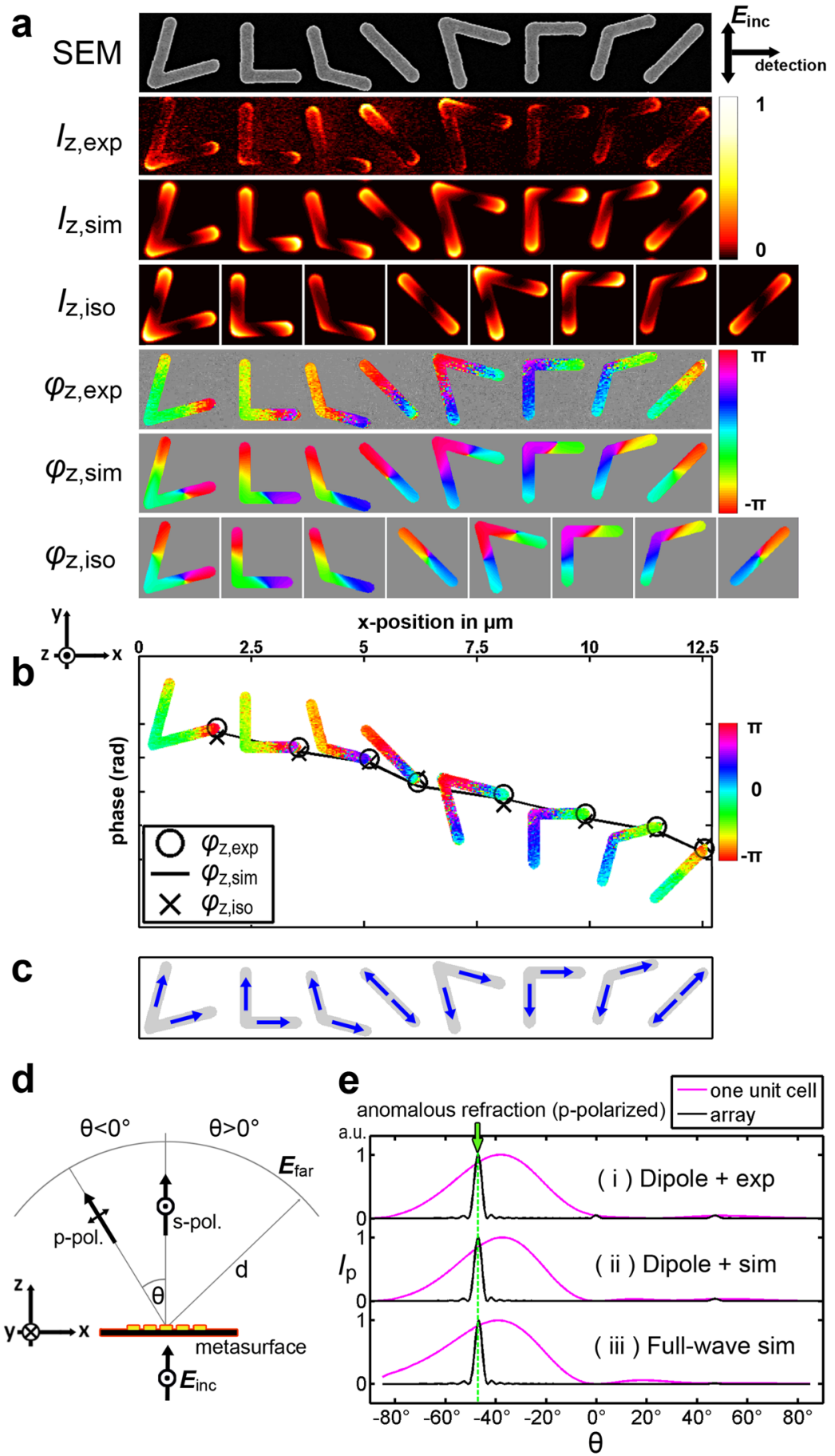

Figure 5. (a) Experimental (exp) and simulated (sim) near-field maps for the unit cell $\left(\Gamma_{x}=12.7 \mu \mathrm{m} \times \Gamma_{y}=1.74 \mu \mathrm{m}\right)$ of a metasurface antenna array that introduces a phase gradient of $-2 \pi / \Gamma_{x}$ to the incident light which is polarized to excite both antenna modes with the same field component: scanning electron microscope (SEM) images, near-field intensity and phase for the $z$-oriented electric field component. Eight additional simulations were performed treating each of these $\mathrm{V}$-antennas individually in an isolated environment (iso). (b) Extracted near-field phase at the right-most tip of the V-antennas from (a), revealing a near-field phase gradient across the unit cell. Circles mark the experimental near-field phase $\varphi_{\mathrm{z} \text {,exp }}$ and the position for each of the eight data points; the solid line shows the data for the simulated near-field phase $\varphi_{\mathrm{z} \text {,sim }}$. The crosses in the plot mark the nearfield phase values $\varphi_{z, \text { iso }}$ above the right-most tip for the respective isolated antennas. (c) Dipole model to represent the unit cell. Each $\mathrm{V}$-antenna is approximated by two dipoles in the center of both arms. The amplitude and phase values for each dipole are taken in the near-field data of (a) at the respective tip of the $\mathrm{V}$-antenna. (d) Schematic side view of the experiment demonstrating the generalized law of refraction. ${ }^{3}$ The incident $y$-polarized light hits the metasurface from below at normal incidence. The s-polarized part of the scattered light continues along the surface normal (normal refraction), whereas the p-polarized part is scattered away from the surface normal at an angle $\theta$ (anomalous refraction). (e) Plots of the intensity $I_{\mathrm{p}}$ (p-polarized) in the far field vs $\theta$ : We plot the far field for one unit cell (pink) and for a $200 \mu \mathrm{m} \times 200 \mu \mathrm{m}$ array (black) using the dipole model with the experimental (i) and simulation (ii) near-field data and compare it to the far-field projection from full-wave simulations (iii). The green arrow shows the predicted angle $\theta_{\text {theo }}$ according to the generalized law of refraction. 
amount of coupling between nearby antennas (see Supporting Information, Sections 2 and 3 for more details). Otherwise the experimental and simulated data sets match well, though we observe a small shift of the average intensity $I_{z, a v g}$ for both modes such that the experimental values $I_{z, \text { avg,exp }}$ are shifted toward the bigger antennas in relation to the simulated $I_{\mathrm{z}, \mathrm{avg}, \mathrm{sim}}$. In terms of a wavelength-dependent intensity distribution $I_{z, \text { avg }}(\lambda)$ for a fixed antenna size this would imply a blue-shift of the experimental data $I_{z \text {,avg,exp }}(\lambda)$ with respect to the simulation values $I_{\mathrm{z}, \mathrm{avg}, \text { sim }}(\lambda)$. We have been unable to determine the origin of this shift; after ruling out the effects of the native silicon oxide on our substrate as well as coupling to neighboring antennas via simulations and double-checking all geometrical parameters of our fabricated samples with SEM and AFM, we present the results as they are.

On basis of these isolated V-antennas, we examined the collective near-field behavior of antenna arrays. We focused on an array which is essentially identical to the one used in previous experiments demonstrating anomalous refraction and reflection, ${ }^{3,22,23}$ but with $\mathrm{V}$-antenna sizes rescaled for our operating wavelength $\lambda=9.3 \mu \mathrm{m}$. As shown in Figure $4 \mathrm{~b}$, the phase response of a single antenna mode can roughly vary between 0 and $\pi$ depending on the antenna size, so for full control of the wavefront (i.e., 0 to $2 \pi$ ) both antenna modes have to be utilized. As in previous experiments, ${ }^{3,22}$ we align this unit cell such that the symmetric and the antisymmetric modes are both excited by the y-polarized incident light for all of the antennas. The unit cell of this metasurface contains eight Vantennas (Figure 5) which were designed to introduce a constant phase gradient $-2 \pi / \Gamma_{x}$ along the $x$-axis to the light scattered in cross-polarization, where $\Gamma_{x}$ is the length of the unit cell in this direction, along with a uniform scattering amplitude. ${ }^{2,3,5,8}$ The symmetry axis $\hat{\mathbf{s}}$ of the first four antennas of the unit cell is oriented along the $45^{\circ}$ diagonal between the $x$ - and $y$-axes, and their opening angles are $\Delta=60^{\circ}, 90^{\circ}, 120^{\circ}$, and $180^{\circ}$, the same angles we examined for the isolated $\mathrm{V}$ antennas above (Figures 1-4). The second four antennas are copies of the first four, but rotated clockwise by $90^{\circ}$ (Figure 5). Our simulations assumed a periodic, infinitely large metasurface, whereas in the experiments the array dimension is 2 periods along $x$ and 6 periods along $y$. The s-SNOM maps are taken at the center of this array.

Figure 5a shows the experimental and calculated near-field intensity and phase maps for a unit cell with $\Gamma_{x}=12.7 \mu \mathrm{m}$ (see Supporting Information, Figure S7 for two additional experimental and simulated data sets with $\Gamma_{x}=15.1$ and 17.4 $\mu \mathrm{m})$.

In Figure 5a, we observe asymmetric near-field patterns on each antenna of the unit cell, indicating that both the symmetric and antisymmetric antenna modes are excited. Furthermore, the near-field phase images now show gradients along the antenna arms rather than abrupt phase jumps as in Figure $4 c, d$, which is characteristic of the simultaneous excitation of several antenna modes. ${ }^{19}$ Note that Figure 5a shows the unit cell with the highest packing density (the smallest value for $\Gamma_{x}$ ), where the eight antennas are very close to each other. This proximity could yield near-field coupling effects that potentially perturb the antenna modes. ${ }^{3}$ To estimate the strength of this coupling, we simulated each of the eight $\mathrm{V}$-antennas independently with the same incident polarization in an isolated environment. The results for the near-field maps in Figure 5a match, with only minor differences between the unit cell in the array (exp and sim) and the isolated antennas (iso). For a larger unit cell length $\Gamma_{x}$, where less near-field coupling is expected a priori, we find even closer agreement with the isolated antennas (see Supporting Information, Figure S7 for larger values of $\Gamma_{x}$ ). Since we are interested in the $x$-polarized scattered light by each element, we analyzed the electron oscillations induced in the antennas along the $x$-direction. Intuitively the flow of charges along $x$ (i.e., from left to right) can be monitored by looking at the field variations at one extreme of the antenna along the $x$-axis. In Figure $5 b$ we chose to compare our experimental and computational results for the near-field phase $\left(\varphi_{\mathrm{z}, \exp }\right.$ and $\left.\varphi_{\mathrm{z}, \mathrm{sim}}\right)$ to the phase of simulated isolated antennas $\varphi_{\text {z,iso }}$ at the right-most (largest $x$ ) point on each antenna, and find little influence of the nearby antennas for even the highest packing density. We conclude that near-field coupling in our metasurfaces is present but minimal, thus not perturbing significantly the designed scattering amplitude and phases of the individual antennas.

The expected far-field response of this metasurfacerefracting normally incident light to an angle away from the normal $\theta_{\text {theo }}=\sin ^{-1}(\lambda / 2 \pi \mathrm{d} \Phi / \mathrm{d} x)$ where $\mathrm{d} \Phi / \mathrm{d} x=-2 \pi / \Gamma_{x}$ is the imparted phase gradient-has been well-established in previous experiments. ${ }^{3,22,23}$ The near-field phase image resolves this constant phase gradient among the eight antennas comprising the unit cell; i.e., we find a $2 \pi$ phase shift across the unit cell with the phase incrementing linearly by approximately $\pi / 4$ for each consecutive antenna (Figure $5 b$ ).

To firmly establish the connection between the near field and the far field, we modeled each of the eight $\mathrm{V}$-antennas as a set of two radiating dipoles, with each dipole replacing one of the antenna arms (Figure 5c). The phase and amplitude for each dipole is assumed to be, to first approximation, the phase and amplitude of the near field at the arm end (tip), where the fields are largest. This model allows us to calculate the far field of a single unit cell by summing the individual contributions of these 16 dipoles. By periodically repeating this unit cell, we obtain the refraction angle provided by a fully illuminated metasurface of size $200 \mu \mathrm{m} \times 200 \mu \mathrm{m}$ (see Methods section for details). As in the previous experiment by $\mathrm{Yu}$ et al. ${ }^{3}$ we distinguish between the normal and anomalous refraction (Figure 5d). The anomalously refracted (p-polarized) component of the far field is plotted in Figure 5e. All three methods used to compute the far fields (the dipole model with experimental near-field data, the model with simulated nearfield data, and the simulated far field obtained without the dipole model) agree well. We observe that the array scatters precisely at the angle $\theta_{\text {theo }}=\sin ^{-1}\left(-\lambda / \Gamma_{x}\right)=-47^{\circ}$ predicted by the generalized law of refraction (shown by green arrow in Figure 5e). ${ }^{3}$ For comparison, we also calculated the radiation patterns for the metasurfaces with periodicities $\Gamma_{x}=15.1$ and $17.4 \mu \mathrm{m}$; see Supporting Information Figure S8.

Note in Figure 5e that the scattered p-polarized field from one single unit cell of the array $I_{\mathrm{p}, \text { unit }}(\theta)$ is distributed over a range of angles for all of the three shown methods (pink line, full width at half $\max \approx 38^{\circ}$ ), while the scattered field from the array $I_{\mathrm{p} \text {,array }}(\theta)$ emerges at only a narrow distribution around $\theta_{\text {array }}$ (black line). Furthermore, the angle of the scattering peak using just one unit cell is at $\theta_{\text {unit }} \approx-37.5^{\circ}$, slightly off from the angle predicted by the generalized law of refraction. Despite this mismatch, the large array scatters at the angle $\theta_{\text {theo }}$ determined by this law. To understand this, we observe that our structure can be likened to a blazed grating ${ }^{24}$ (compared to a conventional blazed grating, our structure is ultrathin, flat, and provides polarization conversion) where the phase gradient 
imparted by the single unit cell of the array provides the "blaze". Though $\theta_{\text {unit }}$ is not precisely equal to $\theta_{\text {array, }}$ the blaze selects a single diffraction order of the array which beams light at precisely $\theta_{\text {theo }}$. For this reason such metasurfaces are robust to small errors in design or fabrication of the individual antennas.

The ability to connect near-field and far-field behavior of antenna arrays is significant not only because it enhances our understanding of metasurfaces, but also because it suggests that near-field measurements such as phase-resolved s-SNOM can be used to predict the corresponding far-field radiation patterns of a wide variety of optical structures. In full-wave electromagnetic simulations, complete information about near fields is commonly used to predict far fields via so-called "far-field transformations", ${ }^{25}$ which are made possible by the surface equivalence principle. $^{26}$ These transformations require knowledge of the full vectorial near field along some surface. In experiments, however, the near-field information is almost always limited; for example, the present s-SNOM experiment only provides information about the z-component of the electric field. Despite this limited information, we demonstrated here that the far-field response can still be predicted. We explain this finding by the fact that for thin antenna structures the z-component of the complex near field is a good proxy for the time-varying charge distribution in the antennas and hence their far-field scattering properties. While the dipole model presented here already yields good prediction of the far field, we envision more sophisticated models where the near field at each position on the antenna surface is taken into account. Such limited-information far-field transformations are worth exploring further in future studies.

We demonstrated near-field imaging of phase gradients in optical metasurfaces based on plasmonic V-shaped antennas. By applying phase-resolved scattering-type scanning near-field optical microscopy (s-SNOM) at infrared wavelengths, we measured the oscillating charge distributions in individual $\mathrm{V}$ shaped antennas and in metasurfaces comprising closely packed antennas, obtaining results in close agreement with full-wave simulations. The near-field images showed that tailoring the size and geometry of the antennas determines their amplitude and phase response, confirming the mechanism for achieving optical phase discontinuities. By combining the SNOM measurements with a model based on oscillating dipoles, we connected the near- and far fields of phased antenna metasurfaces, finding that the far-field response can be inferred from limited, experimentally obtained knowledge of the near fields. This study paves the way for experimental near-field characterization of a large variety of metasurfaces based on metal antennas and a prediction of their far fields from nearfield measurements.

Methods. Fabrication. The antennas were fabricated on high resistivity $(>10000 \Omega \mathrm{cm})$ double side polished silicon using a conventional electron beam (e-beam) lithography process with lift-off. A double layer of poly(methyl methacrylate) (PMMA) resist (495A4, then 950A2; MicroChem) was spun at $4000 \mathrm{rpm}$ onto the silicon wafer, baked at $180{ }^{\circ} \mathrm{C}$ for $3 \mathrm{~min}$, and then exposed using a $100 \mathrm{kV}$ e-beam system (Elionix ELS-F125). After development with 3:1 isopropanol-methyl isobutyl ketone (IPA-MIBK), a $5 \mathrm{~nm}$ titanium adhesion layer and $45 \mathrm{~nm}$ of gold were deposited using e-beam evaporation, and the lift-off process was completed in acetone with ultrasonic agitation. For SEM images of the resulting structures, see Figures 2, 3, and 5.
s-SNOM Measurements. Near-field microscopy setup: we apply interferometric scattering-type scanning near-field optical microscopy (s-SNOM $)^{27-34}$ to map our antenna structures. Our s-SNOM setup is based on an atomic force microscope (AFM), where commercial silicon tips (NanoWorld, ArrowNRC-50) are used to locally scatter the antenna near field. ${ }^{14,35,19,20,29,34}$ The sample and tip were illuminated from below at normal incidence with a weakly focused $\mathrm{CO}_{2}$ laser beam at $\lambda=9.3 \mu \mathrm{m}$ (transmission-mode s-SNOM ${ }^{19,20,35}$ ), thus ensuring homogeneous illumination of a relatively large area on the sample (i.e., covering each complete series of antennas in Figures 2-5) without phase-retardation effects. This illumination closely approximated a plane wave, matching the experimental conditions in the original far-field experiments. ${ }^{3}$

In all of the presented experiments the illuminating beam propagated in the positive $z$-direction and its polarization was fixed along the $y$-axis. Excitation of the symmetric mode for the isolated $\mathrm{V}$-antennas in Figure 2 was achieved by aligning the Vantennas' symmetry axis $\hat{\mathbf{s}}$ along the $y$-direction. Likewise, excitation of the antisymmetric mode in Figure 3 was achieved by aligning the axis $\hat{\mathbf{a}}$ along the $y$-direction. To avoid sample rotation between the measurements, we fabricated two sets of $\mathrm{V}$-antennas on the same substrate where the individual antennas of the second set (antisymmetric mode, Figure 3) were rotated by $90^{\circ}$ with respect to the antennas of the first set (symmetric mode, Figure 2).

Using a parabolic mirror we collected the tip-scattered light in the $x$-direction at an angle of $60^{\circ}$ from the surface normal. The tip-scattered near field was interfered with a phasemodulated, vertically $(z-)$ polarized reference beam at the infrared detector and recorded simultaneously with the sample topography. Background contributions could be fully suppressed by vertical tip oscillation at a frequency $\Omega=250 \mathrm{kHz}$ (tapping-mode AFM) and by subsequent higher harmonic demodulation of the detector signal at $3 \Omega .^{21}$ In this case, the vertical polarization of the reference beam selected the $\mathrm{p}$ component of the tip-scattered light, which mainly contains the vertical ( $\mathrm{z}-$ ) component of the antenna near fields. ${ }^{19,20,35}$ Using this pseudoheterodyne detection module (www.neaspec.com) the amplitude $\left|E_{z}\right|$ and phase $\varphi_{z}$ of the vertical near-field component $E_{z}$ were measured for each scanning point. The near-field intensity is then easily calculated by $I_{z}=\left|E_{z}\right|^{2}$.

Note that it has been found recently that there are different scattering mechanisms in s-SNOM imaging of metal antennas: ${ }^{15,36}$ (i) direct scattering of the antenna near fields by the tip into the far field (detector) and (ii) scattering of the antenna near fields by the tip via the antenna itself. When imaging resonant linear antennas, excitation with s-polarization and detecting the s-component of the scattered light is mainly based on mechanism (ii), while detecting the p-component is mainly based on mechanism (i), where the latter was found to essentially yield the $z$-component, $E_{z}$, of the near fields on the antenna surface. ${ }^{36}$ In our case we aligned the $\mathrm{V}$-antennas such (see Figure $1 \mathrm{~b}$ ) that the excited dipole moment radiates with spolarization in direction of the detection. In this configuration, the p-component of the scattered light is mainly based on mechanism (i) and we essentially detect the vertical $(z-)$ component of the local near fields, which is furthermore supported by the good agreement of the experimental images with the simulations in Figures 2-5.

Simulations. The FDTD simulations were performed using "FDTD Solutions" from Lumerical running on a workstation. For Figures 2 and 3 (also the 55 isolated antennas in the 
Supporting Information, Section 1) and the isolated V-antennas of Figure 5, the simulation of each $\mathrm{V}$-antenna was done inside a volume of $6.2 \mu \mathrm{m} \times 6.2 \mu \mathrm{m} \times 1.5 \mu \mathrm{m}$ using a total-field scattered-field (TFSF) plane wave source $(\lambda=9.3 \mu \mathrm{m})$ with perfectly matched layer (PML) boundary conditions. In contrast to the experiment these $\mathrm{V}$-antennas are truly isolated since there are no excited antennas nearby, which could influence each other.

The simulations for the three antenna arrays (Figure 5a and Supporting Information, Figure S7) use periodic boundary conditions to define a single repeating unit cell with eight antennas in it. A plane wave $(\lambda=9.3 \mu \mathrm{m})$ was launched from the silicon side to illuminate this antenna array. So, we simulate an infinite array whereas in the experiment we measure in the middle of an array of limited size. In all of our simulations we neglect the influence of the cantilever with the silicon tip and the roughness of the gold surface. The relevant monitor (frequency-domain field and power monitor) for the near field was located at a height of $40 \mathrm{~nm}$ above the antenna surface where we obtained a good match with the experimental nearfield images. ${ }^{19,20}$ We took the complex refractive indices given by Palik ${ }^{39}$ for $\mathrm{Ti}, \mathrm{Au}$ and $\mathrm{Si}$.

Visualization and Analysis of the Near Field. The visualization and analysis of experimental and simulated data was performed using Matlab. We used the intensity and phase of the complex near-field component $E_{z}$. For visual comparison we plot the intensity $I_{z}=\left|E_{z}\right|^{2}$ and phase $\varphi_{z}=\arg \left(E_{z}\right)$ for all of the $\mathrm{V}$-antennas (compare to Figures 2 and 3 ). The colorbar for the intensity maps in Figures 2, 3, and 5 is stretched to show the whole series of resonant and nonresonant antennas in one colorscale; this was not needed in Figure 4 where just one Vantenna is shown and the conventional "hot" colorscale was used. In order to analyze the near-field response of each isolated antenna we took two physical quantities for each antenna. First we calculated one distinct average intensity value $I_{\mathrm{z}, \mathrm{avg}}$ for each antenna, which is the sum of all the single intensity data points above the actual antenna position divided by a unitless factor referring to the top-surface area of the respective $\mathrm{V}$-antenna. Then, for each $\mathrm{V}$-antenna series of 11 antennas the average intensity was normalized to unity (Figure $4 a)$. The phase value was measured as the second quantity. For the analysis of Figure $4 \mathrm{~b}$ we defined the phase as the phase value of that point of the left $\mathrm{V}$-antenna tip, where the intensity reaches its maximum. These are data points with a strong signal and thus reliable phase values. Then all of the phase values in each antenna series have been shifted up or down by a constant factor so that the phase value equals $\pi / 2$ at the peak of the $I_{z, \text { avg }}$ graph (compare to Figure 4).

Calculation of the Far Field. Our model treats each arm of the $\mathrm{V}$-antenna as a radiating dipole. These two dipoles are oriented parallel to the arms they replace and are located in the center of the respective arms. The phase and amplitude for each dipole are extracted at the arm end (tip). Then for each of these radiating dipoles $\boldsymbol{p}$ we can calculate the exact electric field $E_{\text {dip }}(r, p)$ in air at a given position $r$ in the far field. ${ }^{38,39}$ In this approximation we neglect the silicon substrate. ${ }^{40}$

We found the far field by calculating the electric field on a semicircle in the $x z$-plane above the metasurface with a radius of $d=1 \mathrm{~m}$ (Figure $5 \mathrm{~d}$ ). For each position on this circle in the far field the total electric field scattered by one unit cell can be calculated using the superposition principle by summing up the fields created by the 16 dipoles of this unit cell, $\boldsymbol{E}_{\text {unit }}(\boldsymbol{r})=$ $\sum_{i=1}^{16} E_{\text {dip }}\left(r-r_{i} p_{i}\right)$ where $r_{i}$ is the position of the $i$-th dipole in the unit cell. To model a finite-size incident beam, we sum $\boldsymbol{E}_{\text {unit }}$ over of 16 unit cells along $x$ and 115 cells along $y$, to obtain an array with the size of about $200 \mu \mathrm{m} \times 200 \mu \mathrm{m}$. This way using all of the dipoles we calculate the total electric field scattered by the metasurface $\boldsymbol{E}_{\text {array }}(\boldsymbol{r})=\sum_{n_{v} n_{y}} \boldsymbol{E}_{\text {unit }}\left(\boldsymbol{r}_{\boldsymbol{u}}\left(n_{x}, n_{y}\right)\right)$, with $\boldsymbol{r}_{\boldsymbol{u}}$ being the center of the unit cell $\left(n_{x}, n_{y}\right)$.

\section{ASSOCIATED CONTENT}

\section{S Supporting Information}

Experimental and simulation data for V-antenna types not shown in the main text. Near-field maps and far-field calculations for metasurfaces with various periods. Comparison of the average intensity between different series of isolated antennas and more information on coupling between antennas. Effect of the antenna thickness on the resonance condition. The Supporting Information is available free of charge on the ACS Publications website at DOI: 10.1021/acs.nanolett.5b00692.

\section{AUTHOR INFORMATION}

\section{Corresponding Author}

*E-mail: mkats@wisc.edu.

\section{Present Address}

F.A.: Hewlett-Packard Laboratories, Palo Alto, California 94304, USA

\section{Author Contributions}

B.J.B. and M.S. contributed equally.

\section{Notes}

The authors declare the following competing financial interest(s): R.H. is co-founder of Neaspec GmbH, a company producing scattering-type scanning near-field optical microscope systems. All other authors declare no competing financial interests.

\section{ACKNOWLEDGMENTS}

The authors acknowledge helpful discussions with N. Yu, P. Genevet, and Z. Gaburro. F.C. acknowledges financial support from the Air Force Office of Scientific Research under grant number FA9550-12-1-0289 and from Draper Laboratory. M.S. and R.H. acknowledge financial support by the ERC Starting Grant No. 258461 (TERATOMO) and by the National Project MAT2012-36580 from the Spanish Ministry of Economy and Competitiveness. The samples were fabricated in the Center for Nanoscale Systems at Harvard University, which is a member of the National Nanotechnology Infrastructure Network supported by the National Science Foundation.

\section{REFERENCES}

(1) Yu, N.; Genevet, P.; Aieta, F.; Kats, M. A.; Blanchard, R.; Aoust, G.; Tetienne, J.-P.; Gaburro, Z.; Capasso, F. IEEE J. Quantum Electron. 2013, 19, 4700423.

(2) Yu, N.; Capasso, F. Nat. Mater. 2014, 13, 139-150.

(3) Yu, N.; Genevet, P.; Kats, M. A.; Aieta, F.; Tetienne, J.-P.; Capasso, F.; Gaburro, Z. Science 2011, 334, 333-337.

(4) Sun, S.; He, Q.; Xiao, S.; Xu, Q.; Li, X.; Zhou, L. Nat. Mater. 2012, 11, 426-431.

(5) Aieta, F.; Genevet, P.; Kats, M. A.; Yu, N.; Blanchard, R.; Gaburro, Z.; Capasso, F. Nano Lett. 2012, 12, 4932-4936.

(6) Ni, X.; Ishii, S.; Kildishev, A. V.; Shalaev, V. M. Light: Sci. Appl. 2013, 2, e72.

(7) Chen, X.; Huang, L.; Mühlenbernd, H.; Li, G.; Bai, B.; Tan, Q.; Jin, G.; Qiu, C.-W.; Zhang, S.; Zentgraf, T. Nat. Commun. 2012, 3, 1198 . 
(8) Genevet, P.; Yu, N.; Aieta, F.; Lin, J.; Kats, M. A.; Blanchard, R.; Scully, M. O.; Gaburro, Z.; Capasso, F. Appl. Phys. Lett. 2012, 100, 013101.

(9) Yu, N.; Aieta, F.; Genevet, P.; Kats, M. A.; Gaburro, Z.; Capasso, F. Nano Lett. 2012, 12, 6328-6333.

(10) Kats, M. A.; Genevet, P.; Aoust, G.; Yu, N.; Blanchard, R.; Aieta, F.; Gaburro, Z.; Capasso, F. Proc. Natl. Acad. Sci. U.S.A. 2012, 109, 12364-12368.

(11) Pfeiffer, C.; Grbic, A. Phys. Rev. Lett. 2013, 110, 197401.

(12) Zhao, Y.; Alu, A. Phys. Rev. B: Condens. Matter Mater. Phys. 2011, 84, 205428.

(13) Kats, M. A.; Yu, N.; Genevet, P.; Gaburro, Z.; Capasso, F. Opt. Express 2011, 19, 21748-21753.

(14) Dorfmüller, J.; Vogelgesang, R.; Weitz, R. T.; Rockstuhl, C.; Etrich, C.; Pertsch, T.; Lederer, F.; Kern, K. Nano Lett. 2009, 9, 23722377.

(15) Alonso-González, P.; Albella, P.; Schnell, M.; Chen, J.; Huth, F.; Garcia-Etxarri, A.; Casanova, F.; Golmar, F.; Arzubiaga, L.; Hueso, L. E.; Aizpurua, J.; Hillenbrand, R. Nat. Commun. 2012, 3, 684.

(16) Dorfmuller, J.; Vogelgesang, R.; Khunsin, W.; Rockstuhl, C.; Etrich, C.; Kern, K. Nano Lett. 2010, 10, 3596-3603.

(17) Alonso-González, P.; Albella, P.; Neubrech, F.; Huck, C.; Chen, J.; Golmar, F.; Casanova, F.; Hueso, L. E.; Pucci, A.; Aizpurua, J.; Hillenbrand, R. Phys. Rev. Lett. 2013, 110, 203902.

(18) Novotny, L. Phys. Rev. Lett. 2007, 98, 266802.

(19) Schnell, M.; Garcia-Etxarri, A.; Huber, A. J.; Crozier, K.; Aizpurua, J.; Hillenbrand, R. Nat. Photonics 2009, 3, 287-291.

(20) Schnell, M.; Garcia-Etxarri, A.; Alkorta, J.; Aizpurua, J.; Hillenbrand, R. Nano Lett. 2010, 10, 3524-3528.

(21) Ocelic, N.; Huber, A.; Hillenbrand, R. Appl. Phys. Lett. 2006, 89, 101124.

(22) Aieta, F.; Genevet, P.; Yu, N.; Kats, M. A.; Gaburro, Z.; Capasso, F. Nano Lett. 2012, 12, 1702-1706.

(23) Ni, X.; Emani, N. K.; Kildish, A. V.; Boltasseva, A.; Shalaev, V. M. Science 2012, 335, 427-427.

(24) Larouche, S.; Smith, D. R. Opt. Lett. 2012, 37, 2391-2393.

(25) Barth, M. J.; McLeod, R. R.; Ziolkowski, R. W. J. Electromagn. Waves Appl. 1992, 6, 5-18.

(26) Balanis, C. A. Advanced engineering electromagnetics; Wiley: New York, 1989.

(27) Hillenbrand, R.; Keilmann, F. Appl. Phys. B: Lasers Opt. 2001, 73, 239-243.

(28) Hillenbrand, R.; Keilmann, F.; Hanarp, P.; Sutherland, D. S.; Aizpurua, J. Appl. Phys. Lett. 2003, 83, 368-370.

(29) Esteban, R.; Vogelgesang, R.; Dorfmuller, J.; Dmitriev, A.; Rockstuhl, C.; Etrich, C.; Kern, K. Nano Lett. 2008, 8, 3155-3159.

(30) Olmon, R. L.; Krenz, P. M.; Jones, A. C.; Boreman, G. D.; Raschke, M. B. Opt. Express 2008, 16, 20295-20305.

(31) Kim, Z. H.; Leone, S. R. Opt. Express 2008, 16, 1733-1741.

(32) Kim, D.-S.; Heo, J.; Ahn, S.-H.; Han, S. W.; Yun, W. S.; Kim, Z. H. Nano Lett. 2009, 9, 3619-3625.

(33) Kim, D.-S.; Kim, Z. H. J. Korean Phys. Soc. 2008, 52, 17-20.

(34) Rang, M.; Jones, A. C.; Zhou, F.; Li, Z.-Y.; Wiley, B. J.; Xia, Y.; Raschke, M. B. Nano Lett. 2008, 8, 3357-3363.

(35) Schnell, M.; Garcia-Etxarri, A.; Huber, A. J.; Crozier, K. B.; Borisov, A.; Aizpurua, J.; Hillenbrand, R. J. Phys. Chem. C 2010, 114, 7341-7345.

(36) Neuman, T. Mapping the near-fields of plasmonic nanoantennas by scattering-type Scanning Near-field Optical Microscopy. Submitted to Laser Photonics Rev.

(37) Palik, E. D. Handbook of optical constants of solids; Academic Press: San Diego, 1998.

(38) Jackson, J. D. Classical electrodynamics, Vol. 3; Wiley: New York, 1962.

(39) Tetienne, J.-P.; Blanchard, R.; Yu, N.; Genevet, P.; Kats, M. A.; Fan, J. A.; Edamura, T.; Furuta, S.; Yamanishi, M.; Capasso, F. New J. Phys. 2011, 13, 053057.
(40) Blanchard, R.; Aoust, G.; Genevet, P.; Yu, N.; Kats, M. A.; Gaburro, Z.; Capasso, F. Phys. Rev. B: Condens. Matter Mater. Phys. 2012, 85, 155457. 